\title{
Researches on the Progress of Antiallergic Traditional Chinese Medicine and Its Mechanism
}

\author{
Xuan WU* \\ Jiangxi University of Traditional Chinese medicine, \\ NO.18 Yunwan Road, Wanli District, Nangchang, China \\ ${ }^{*}$ Corresponding author
}

Keywords: Traditional Chinese medicine, Allergy, Mechanism.

\begin{abstract}
With the study of anti-allergy medicine, it proves traditional Chinese medicine has anti-allergic effects and several other functions, such as protecting and stabilizing the target cell membrane (reduce or prevent degranulation, release the allergy mediators, increase the intracellular cAMP levels), inhibiting the formation of $\mathrm{IgE}$, against allergies media, neutralizing the allergen and so on. In addition, it has good clinical effects and no significant adverse reactions. This paper take reference to a variety of literatures and introduces the anti-allergic effect of single herb and compound traditional Chinese medicine. On the basis of it, we compared the advantages of the traditional Chinese medicine with the western medicine in the treatment of allergy.
\end{abstract}

\section{Anti-allergic Effects of the Single Herb Traditional Chinese Medicine}

\section{Conidium}

Conidium always be used for the treatment of skin eczema. And the pharmacological experiments shows that the Conidium coumarin extracted from the snake bed has the significant anti-allergic effects, Conidium coumarin in vitriol can resistance the histamine. And slow-reacting substance has the inhibition effects on the contraction of tracheal and smooth muscle in guinea pig and the passive cutaneous anaphylaxis in mice.

\section{Leaves}

Washing with the soup of the Leaves can treat the skin eczema, and leaves oil plays a role in anti-allergic, cough, phlegm, asthma. Leaves oil capsules has treated 44 cases of allergic diseases such as allergic asthma, purpura, dermatitis, drug eruption and other allergic diseases, and the total effective rate was $77.3 \%$. Counts the blood basophils of seven asthma cases before and after the treatment. Then, from which they can see that it is generally low before treatment, the range is $11--33 / \mathrm{mm}^{3}$, the mean is $22.1 / \mathrm{mm}^{3}$, However, it is generally increased after treatment, and the range is $22--10 / \mathrm{mm}^{3}$, the mean is $50.3 / \mathrm{mm}^{3}$.

\section{Flom Magnolias}

Its volatile oil significantly inhibited the HA and Ach-induced contraction of guinea pig ileum, and significantly inhibit anaphylactic contraction of guinea pig ileum. Moreover, its volatile oil can prevent the mast cell degranulation of the rats.

\section{Tripterous}

Its total glycosides can inhibit the antigen-specific antibody cells and antibody-secreting cells to produce the anti-sheep red blood cells, the old tuberculin 
delayed hypersensitivity in guinea pigs, and the immune rosette to converted to lymphocyte transformation. Moreover, it can significantly inhibit the peripheral mononuclear cells to produce total IgM -RF in vitriol of the rheumatoid arthritis patients.

\section{Jujube}

Its ethanol extract have the same function with immunosuppressant azathioprine, which is inhibited the rats' antibodies reactive.

\section{Licorice}

There are anti-allergic effect of glycerinate, and the glycerinate can prolong the survival time of transplanted tissue, inhibit the macrophages to intake the B. Subtilist $\alpha$-amylase (BA), and significantly inhibit the guinea pig skin reactions caused by egg and reduce the symptoms of anaphylactic shock.

\section{Ginkgo}

The water-soluble of its Outer seed coating can prevent the sensitized lung tissue of the guinea pig to release the histamine and the SRS-A, inhibit the PCA in mice and skull periosteum mast cell degranulation in rat, and can directly antagonize the contractile response of guinea pig ileum smooth muscle caused by allergies media.

\section{Sophora}

Its effective part Sophora alkaloids (Oxymetazoline) can inhibits the activity of the cyclic nucleotide phosphodiesterase, increase the intracellular cAMP, and prevents mast cells to release histamine. Guinea decoction and spray alkaloids have significantly asthma effects on the Guinea pig asthma caused by the heavy histamine dihydrochloride and the strength is similar to the aminophylline. Chen injects marine to treat eczema in 24 cases, 8 cases cured, improved in 12 cases, invalid 4 cases, the total efficiency is $83.3 \%$, which is better than the Procaine group(overall efficiency is $64 \%)$.

\section{Ganoderma}

Its alcohol precipitation broth antiserum can significantly reduced passively sensitized skin reactions caused by the ovalbumin and tetanus antiserum. And its fermentation and the extract of different parts can inhibit the release of the actively sensitized histamine and slow-reacting substance which can suppress that Ganoderma can inhibit allergic reactions.

\section{Dan}

It has the effects of eliminating the heat, cooling the blood and blood circulation. Animal tests show that the effective monomer substances extracted from Dan Paeonol have the anti-allergy and anti-histamine effect. Using the Paeonol Waicha cream to treat eczema, contact dermatitis, pruritus of 102 cases, the recovery is $46 \%$, improved is $38 \%$, and ineffective is $16 \%$. The total effective rate was $84 \%$.

\section{Walnuts}

Its water extract can inhibit skin irritation in mouse serum antibodies, and its ethanol extract can inhibit the pigment exudation produced by the passive cutaneous anaphylaxis caused by the blood Shaw of mice's anti-allergic skin. These two points proved that the Walnuts have anti-allergic effects. 


\section{Anti-Allergic Effects of Compound Traditional Chinese Medicine Preparation}

\section{Belamcanda and Ephedra Decoction}

From the animal experiments, Tam found and made the conclusion as follows,Firstly, by determining the amount of the induced interleukin -2 (IL-2) in guinea pigs they found that Belamcanda and Ephedra Decoction can significantly promote the production of IL-2. Secondly, the determination of guinea pigs sensitized mast cell degranulation shows that Belamcanda and Ephedra Decoction can effectively prevent the occurrence of type I allergy. Finally, the determination of the total serum IgE levels of the guinea pigs said that the Belamcanda and Ephedra Decoction can inhibit IgE production and prevent the occurrence of type I allergy.

\section{Ephedra Forsythia Red Bean Soup}

From the paper, Zhang found that the parties and their subtraction side (on the basis of the original parties plus Nepeta, wind 15g, Fructus 30g) can significantly inhibit the histamine-induced localized itching of guinea pig and the dextran-induced dust body itching of mouse.

\section{Peony and Licorice Decoction}

From the paper, Qian found that this parties plays a relief role in the histamine-induced bronchial smooth muscle spasm of guinea pig, and could significantly inhibit the number of asthma and degranulation, which can indicate that the Shaoyaogancao soup has anti-allergic effects.

\section{Xiaoqinglong Decoction}

This is a compound containing eight kinds of Traditional Traditional Chinese medicines, and it is proved by the extract experiment that Xiaoqinglongtang can significantly inhibit the passive cutaneous anaphylaxis in guinea pigs caused by egg and egg white anti-IgE antibody. Meanwhile, it can also inhibit the alkali-induced inflammation cause by histamine, serotonin, acetylcholine, and slow reacting substance.

\section{Chai Pak Soup}

The Chai Pak soup consists of 10 kinds of crude drugs like radix and sage. It is proved by pharmacological experiments that: Chai Pak soup can inhibit the oxidation-induced skin contact allergy in mice (IV-type hypersensitivity reactions) and can strongly inhibit the Body type IV hypersensitivity reactions. And The Chai Pak soup has treated 22 cases of asthma in children, treatment for three months, after checking all the patients, they found that all the patients had vary degrees improvement in symptoms after administration, and no side effects.

\section{Large and Small Chaihu Decoction}

Experiments show that these have a strong inhibitory effect on the mast cell degranulation and histamine release. Tips: Radix, ginger, jujube, Pinellia may inhibit mast cell degranulation and histamine release of mice. Which provides a scientific basis for the two parties can treat allergic diseases. Tanizaki has cured steroid-dependent severe refractory asthma 28 cases, 4 cases theyre markedly effective and 14 cases are effective, and the total efficiency is $64.3 \%$. Moreover it has significant effect for the treatment of excessive secretion of asthma and bronchitic obliteration. Mechanism of the action is inhibiting the free histamine and enhanced systemic and local immune function. 


\section{Advantages of the Anti-Allergy Traditional Chinese Medicine}

Compared with western medicine, the advantages of the antiallergic traditional Chinese medicine are as follows:

Firstly, treatment of allergic disease with traditional Chinese medicine has the advantages in treating the symptoms pathogenesis. On the one hand, it can resist to allergic reactions, on the other hand, it can modulate the immune system, such as Stellaria, Liquesce, Buplever and so on. Secondly, Antiallergic traditional Chinese medicine always acts on various aspects of allergic reactions such as increasing the intracellular AMP levels, stabilizing the cell membrane, inhibiting or reducing the release of biologically active substances, neutralizing the antigen and inhibiting the formation of $\operatorname{IgE}$, and so on. Meanwhile, the effects of antiallergic traditional Chinese medicine on the biological activity are often working on a variety of biologically active substances. As Gao reports in 52 kinds of antiallergic traditional Chinese medicine (single taste 43 kinds, compound 9 kinds) there are 42 kinds can resist two or more bioactive substances. Finally, antiallergic traditional Chinese medicine is effective for treating allergic diseases, has no significant adverse reactions and easily accepted by patients. It's commonly used with anti-histamine (H1 receptor) drug to treat allergic disease, such as Chloramine, diphenhydramine, promethazine, cyproheptadine, ketotic and endotoxin. But the patients always be with drowsiness, dry mouth, dizziness and other side effects. So the patients are not willing to accept them. Moreover, if long-term use, it is easy to produce resistance. However, using the antiallergic traditional Chinese medicine is not only effective but also no recurrence after treatment. Compared with western medicine and antiallergic traditional Chinese medicine, we found that antiallergic traditional Chinese medicine has many advantages, and antiallergic traditional Chinese medicine will be the direction of allergy research.

\section{Conclusion}

In summary, there are more and more research of anti-allergy of Traditional Chinese medicine, not only the single herb Traditional Chinese medicine and its compound preparation have obvious anti-allergic activity, the extracts or active ingredients of some Chinese herbal medicine are also proved to be anti-allergic. Combining the advantages of traditional Chinese medicine and western medine will make a breakthrough in the anti-allergic research in the future.

\section{Literature Reference}

[1] Da-hong Zheng, Xiao-mei Fang. Give you a heads--Apiary should be equipped with allergy. [J], China apiculture; 2006 02.(In Chinese)

[2] Li-xiao Li, Yong-hong Zhang. Experimental study of anti-allergic effects of volatile oil. 520Chinese Journal of Hospital Pharmacy, 2002,22 (9): 520. (In Chinese)

[3] Hong-quan Zhang, Li-li Xu, Qiao-xiu Jin, et al.The anti-allergic effects of sarcoptes soluble components . Traditional Traditional Traditional Chinese medicine, 1990,15 (8): 48. (In Chinese) 
[4] Su-shan Qing. Research on anti-allergic effects of tea. International Journal of Traditional Traditional Traditional Chinese medicine Volume, 1995,17 (2): 43. (In Chinese)

[5] Zhe Wang, Xi-ping Gong, Jing Yang, et.al. Anti scattered anti-allergic effect study [J] Pharmacology and Clinics, 2013,05: 92-95. (In Chinese)

[6] Su Huang, Chen-jia Pei, Guang-yi Wang. Bear bile and other traditional Traditional Traditional Chinese medicine magnolia anti-allergic effects [J]. Pharmacology and Clinical,1996,03:28-29. (In Chinese)

[7] Xiao-dong Hua, Chun-hui Yin. Research progress of traditional Traditional Traditional Chinese medicine and its mechanism [J] allergy. Tianjin Pharmaceutical 2009,06:69-72. (In Chinese)

[8] Chun-ling Ji. Medicine Allergy Recent experimental studies and clinical application [J]. Henan University ,2004,04:85-88. (In Chinese)

[9] Huan-huan Liu. Experimental study of anti-allergic effects of Physalis [D] Yanbian University, 2006. (In Chinese)

[10] Rong Sun, Xiao-lu Qian, Li-li Lv. A comparative study about anti-allergic effects of different active ingredients inmagnolia [J]. Chinese pharmacovigi- lance, 2013, 02:71-73. (In Chinese) 